\title{
Photonic-Crystal Demultiplexer With Improved Crosstalk by Second-Order Cavity Filtering
}

\author{
Henri Benisty, Member, IEEE, Cyril Cambournac, Frederik Van Laere, Student Member, IEEE, and \\ Dries Van Thourhout, Member, IEEE
}

\begin{abstract}
In this paper, we implement on a silicon-on-insulator (SoI) platform a photonic-crystal demultiplexer that operates on the principle of mini-stopbands of broader waveguides. Previous InP-based membrane versions showed modest crosstalk at $10 \mathrm{~nm}$ spacing. Here, we implement a second cavity in the form of a second parallel waveguide almost identical to the core one. Simulations employing three-modes coupled-mode theory and finite-difference time-domain help optimizing the coupling. The experimental realization with deep ultraviolet exposure and grating couplers is the first of this demux strategy on SoI. It successfully shows crosstalk in the 15-20 $\mathrm{dB}$ range on a very small footprint.
\end{abstract}

Index Terms-Demultiplexer, mini-stopband, photonic crystal, silicon-on-insulator (SoI), waveguide.

\section{INTRODUCTION}

D EMULTIPLEXING OPTIONS in guided optoelectronics come in two flavors. The first nonresonant one exploits gratings (planar spectrometer) or related arrayed waveguide systems, the so-called "phasar" [1], [2] or the newer superprism versions [3], [4]. The second one exploits the resonances of systems such as microrings [5]-[7] (see [8] for a range of silicon-based realizations) or photonic crystal nanocavities to extract selected channels. The mini-stopband (MSB) demultiplexing option, based on modal anticrossing in a photonic crystal waveguide, was first demonstrated in 2005 [9]. It is based on the "resonant" coupling of the fundamental mode with a higher order, easy to extract, mode that rapidly leaks through the initial guide photonic barrier [see Fig. 1(a)]. It was first implemented in the so-called "substrate" option on InP-based systems [10]-[13]. Then, as a result of a common effort within the European project FUNFOX, a membrane InP implementation was successfully demonstrated [14]-[16], together with compact $(\sim 12 \mu \times 12 \mu)$ grating couplers to address the chip from out-of-plane fibers. This was a success in the sense that an all-photonic-crystal polarization diversity chip could be demonstrated.

Manuscript received October 16, 2009. First published February 22, 2010; current version published March 31, 2010. This work was supported in part by the ePIXfab facility.

H. Benisty and C. Cambournac are with the Laboratoire Charles Fabry de Institut d'Optique, Centre National de la Recherche Scientifique, Université ParisSud, 91127 Palaiseau cedex, France (e-mail: henri.benisty@institutoptique.fr).

F. van Laere was with the Department of Information Technology, Ghent University, 9000 Gent, Belgium. He is now with Arcelor-Mittal, 9042 Gent, Belgium.

D. van Thourhout is with the Department of Information Technology, Ghent University, 9000 Gent, Belgium.

Color versions of one or more of the figures in this paper are available online at http://ieeexplore.ieee.org.

Digital Object Identifier 10.1109/JLT.2010.2043057 (a)

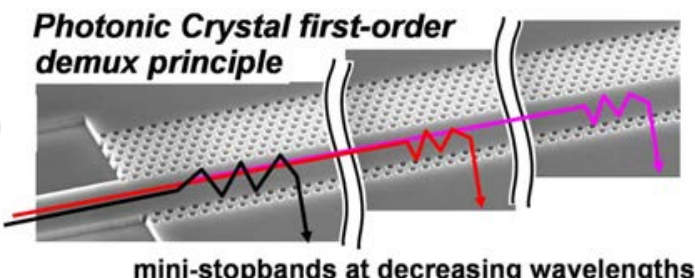

(b)

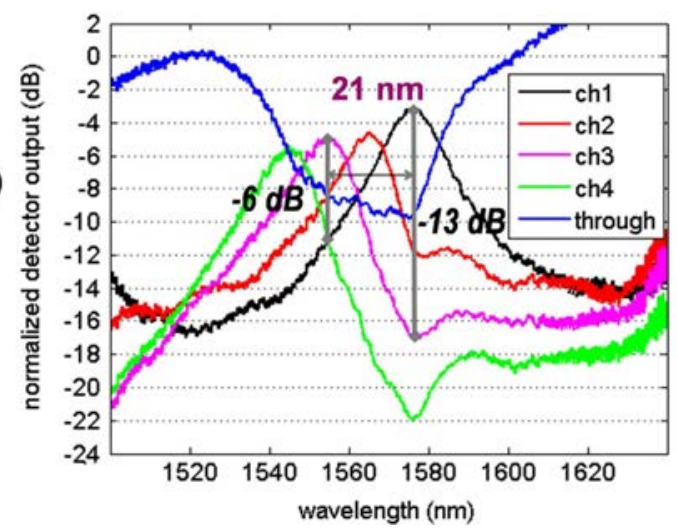

Fig. 1. (a) Photonic crystal MSB demux principle, with a decreasing guide width, adjusted to each channel central wavelength; (b) typical experimental results on a four channel chip made on an InP membrane [14]-[16]. The channel spacing was $10 \mathrm{~nm}$. Note the $-6 \mathrm{~dB}$ typical crosstalk among $10 \mathrm{~nm}$ channels, reaching $13 \mathrm{~dB}$ across the $21 \mathrm{~nm}$ spaced channels ch1 and ch3.

However, a limited crosstalk appeared from experiments as well as from simulations : in the targeted coarse-wavelength-division-multiplexing (CWDM) context, a $10 \mathrm{~nm}$ channel spacing was possible from the lithographic viewpoint (using e-beam lithography to control the reduction of guide width), but the individual resonances were not of flat-top type and thus led to poor crosstalks of the order of 5-13 dB [Fig. 1(b)].

This issue is well known in either type of demultiplexer. For resonant ones, the solution is to cascade resonant elements to go to higher order filter response functions as much as losses allow. A splendid achievement in this respect is the 11th-order microring filter demonstrated by Little et al. [5].

We proposed in [12] and [17] a twin waveguide structure in order to implement the same principle for our photonic crystal waveguide demultiplexing approach, whereby the second waveguide acts as the extra filter element (see Fig. 2). In this paper, we first detail design tricks that have to be applied to take advantage of resonant modes of small but nonzero group velocities. We thus introduce a two-section scheme in each channel, whereby the symmetry of the photonic crystal boundaries in each section 


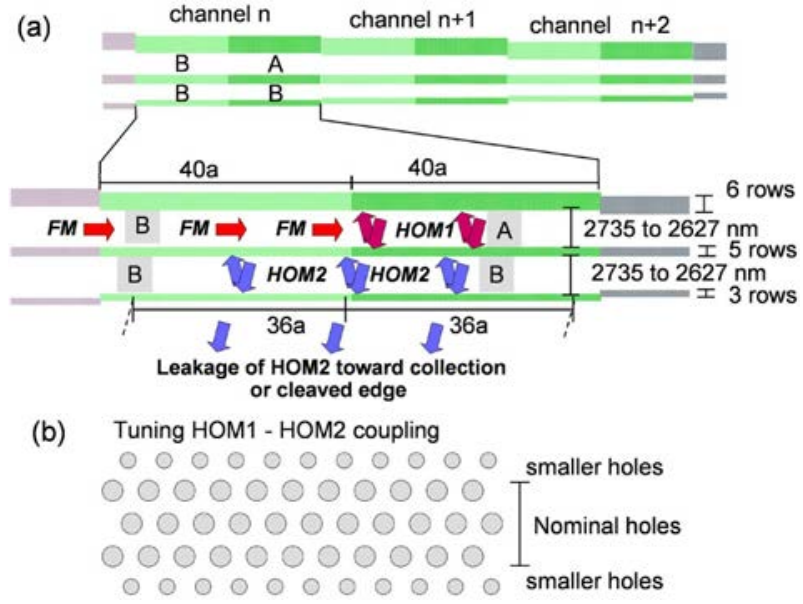

Fig. 2. (a) Principle of the multichannel second-order MSB demux; the FM is converted into HOM1, which is further converted into HOM2, which eventually leaks out. Dimensions reported are those of the experimentally reported structure; (b) scheme of a coupling barrier between HOM1 and HOM2 with smaller holes to tune the coupling strength between values associated to integer rows.

is used to obtain the desired mode couplings and cancel the unwanted ones. The coupled-mode theory that describes the operation of such a two-section channel using three modes is then illustrated, and the compromise between crosstalk and flatter bandpass briefly examined. We then investigate some 2-D finite-difference-time-domain (2D-FDTD) modeling results that give a better view of the crosstalk in a two-channel case, as well as the influence of losses, notably on the spectral channel profile.

As for the technological implementation, we describe it schematically, based on existing similar silicon-on-insulator (SoI) devices realized in the ePIXfab academic foundry silicon facility [8], [18], and on the similar physics of the previous InP membrane realizations [14]-[16]. Finally, the results of selected measurements from an ePIXfab device are given, assessing the improved crosstalk. Overall, this achievement leaves only a reduced gap between laboratory performances and real-world-telecom performances with the provision of not quantified insertion losses, which are briefly discussed.

\section{BACKGROUND}

The MSB is an anticrossing between guided modes that would be uncoupled without the periodicity of the $\mathrm{PhC}$ waveguide edges. This latter couples the modes whose dominant propagation constant differ by $\pi / a, a$ being the lattice constant of the $\mathrm{PhC}$, coinciding with the waveguide periodicity in the preferred case of a triangular lattice of holes with waveguide along the dense row, also known as the $\Gamma \mathrm{K}$ direction. The effects of zone-folding and the applicability of coupled mode theory to this situation has been amply demonstrated in previous work [10], [12], [19]-[21].

The basic result of decoupling the higher order mode through a $\mathrm{PhC}$ barrier without further management is known to lead to excessive crosstalk [14], [15]. Intuitively, the mechanism is a first-order resonance, individually characterized by a resonance shape whose wings are Lorentzian. The crosstalk/bandwidth compromise is then intrinsic: in the multichannel context with given spacing, only higher selectivity can improve crosstalk at the expense of bandwidth.

In our MSB context, the resonance essentially evokes the Fabry-Perot fate of the higher order mode, which "filters" the otherwise smooth and featureless diffraction effect of the fundamental mode skimming the periodic boundaries.

Higher order filtering is the solution currently employed, derived from passive signal filtering, e.g., in electronics. Implementation of multiple microrings is a successful example of how filters are improved, leading to flat-top characteristics and a kind of miniband formation [5], [7].

Thus, implementation of second-order filtering amounts to impose an extra Fabry-Perot resonance adjacent to the one taking place in the first waveguide. Such an option was inserted in [22] in a related context and was, in the present context, pictured in [12] and [17]. These adjacent waveguides are named WG1 (input of signal) and WG2 (filtering). Here, we give a precise design, basic models as result from a first round of optimization, and first corresponding experimental results.

\section{DESIGN AND THEORY}

The MSB is conveniently modeled by coupled mode theory (CMT) as the interaction of the fundamental mode and higher order mode (HOM) of the same waveguide, both with slowly varying envelopes [21]. The demultiplexed signal is the intentional leakage of the HOM mode across a thinned barrier of the photonic crystal waveguide [9]-[12], [14], [15]. The simple duplication of the same waveguide after the barrier would not only lead to an extra resonance, but also to the unwanted channeling of power in the fundamental mode (FM) of this second waveguide. So, in spite of its technological simplicity, which would give fair insurance that both waveguides resonate identically (same frequency, more precisely same dispersion), light would not be clearly extracted sideways. This channel could still be measured, but cascading several filters would then be impossible, as the FM with its good propagation would just feed all signals together to a single port.

To retain sideways extraction and lithographic advantages of waveguide duplication, a trick is to switch the parity of WG2 waveguide edges from "A" to "B", i.e., from even to noneven with respect to guide center, the $\mathrm{B}$ type corresponding to a halfperiod shift between both photonic crystal boundaries [19], [20], [23]. This suffices to cancel the coupling of HOM to FM by symmetry.

Hence, in the filtering WG2 waveguide, the HOM and FM can be resonant (same frequency and wavevector-wise), coupled by periodicity, but the strength of the coupling vanishes for the " $\mathrm{B}$ " arrangement. These properties have been probed in waveguides and demultiplexers in [10], [19], [20], and [23].

This A/B shift, used between WG1 (conversion) and the adjacent WG2 (filtering), can also be used to implement "dead sections" between the channels of the first waveguide WG1. The HOM has a weak, but not zero and variable backward group velocity in convenient cascaded demultiplexer designs. It thus tends to leak back in the feeding line, up to the previous channel. Dead zones of B type, free of HOM/FM interaction can then be used to clean up the HOM by letting it leak sideways, for example, while letting the FM do its way forward to the A section. 
With these effects in mind, the overall design of the present filter takes the form of Fig. 2(a). It involves a two-section B-B/A-B (entrance/conversion sections) arrangement per channel. The B-B "entrance" section serves to clean up or extract HOMs, whereas the primary conversion is made in the A part of the subsequent A-B section, intrinsically filtered by the adjacent B section. All these B sections do not further generate any FM amplitude, especially in the filtering guide (the bottom, B-only one).

Since there is no immediate penalty to lengthen the entrance B-B section, we chose equal length of entrance (B-B) and conversion sections. This relaxes requirements on the low-backward group velocity of the HOM and retains the spirit of a miniature demux.

The main further design elements are then the common length of the A and B sections, and most importantly, the two barrier thicknesses: between both guides, and between second guide and outside (leakage) toward the photodetection stage.

This system locally has three interacting modes: FM1, HOM1, and HOM2 [see Fig. 2(a)]. FM1 and HOM1 are coupled by WG1. HOM1 then coupled to HOM2. Mode HOM2 eventually leaks outside, which is represented by a damping (loss) term. In addition, all modes suffer from some intrinsic waveguide losses that can be taken as inversely proportional to their group velocity. In our design window, HOM's and FM's group velocities typically differ by a factor of 10, depending on the exact channel.

It is intuitive that the second barrier should leak more than the intermediate one: otherwise, light in the two modes HOM1 and HOM2 would be better described as combinations of supermodes HOM1 \pm HOM2. HOM2 could coherently feedback into HOM1 in other words, which obviously spoils the desired channel outcoupled light. It cannot be efficient to have all photons traveling twice across lossy barriers. So, the barriers have to be of different strength, but to finely shape spectra, the final barrier cannot be too weak either, as it would amount to let the A section do the filtering job alone, and bring the result back to first-order filtering and low crosstalk around resonances. So, with still similar barrier strengths, the full coherent picture is needed, precluding the use of the simpler "rate picture." In Fig. 2(b), we anticipate on the realization and present a convenient way to tune the strength of a barrier (namely the central HOM1-HOM2 barrier) by diminishing the hole diameters of edge rows.

We therefore use CMT in the form of the following three equations:

$$
\begin{aligned}
\frac{d F}{d z} & =j\left(n_{\mathrm{g}, \mathrm{F}}^{\mathrm{eff}} / c\right)\left(\omega-\omega_{o}\right) F-\alpha_{F} F+i \kappa_{F H} H_{1} \\
\frac{d H_{1}}{d z} & =j \beta_{H}(\omega) H_{1}-\alpha_{1} H_{1}-i \kappa_{F H} F+i \kappa_{12} H_{2} \\
\frac{d H_{2}}{d z} & =j \beta_{H}(\omega) H_{2}-\alpha_{2} H_{2}-i \kappa_{12} H_{1}
\end{aligned}
$$

where $F$ is the fundamental mode and $\mathrm{H}_{1,2}$ are the HOM1 and HOM2 modes. The dispersion of the FM is linear with group index $n_{\mathrm{g}, \mathrm{F}}^{\mathrm{eff}}$ and attains zero wavevector (in the folded Brillouin zone) at frequency $\omega_{o}$. The HOM uncoupled dispersion $\beta_{H}(\omega)$ is taken as locally quadratic, i.e., $\beta_{H} \sim\left(\omega-\omega_{o, H}\right)^{2}$, with the proper coefficient deduced from standard Fabry-Perot physics and an adjustable frequency at $\beta_{H}=0$. The core anticrossing for conversion thus occurs when $\beta_{H}(\omega)=n_{\mathrm{g}, \mathrm{H}}^{\mathrm{eff}}\left(\omega-\omega_{o}\right)$ for some $\omega>\omega_{o}$. Frequencies may be replaced by the usual dimensionless quantities $u=\omega a / 2 \pi c=a / \lambda_{\mathrm{vac}}$. Losses $\alpha_{F}$, etc., draw power of the different modes, but note that $\alpha_{2}>\alpha_{1}$ because the difference accounts for the desired side leakage. Thus, the local output power at a channel exit is in principle the adequate fraction of $\alpha_{2} H_{2}$, not the whole of it. Finally, $\kappa_{F H}$ is the coefficient coupling FM and HOM1, while $\kappa_{12}$ couples HOM1 and HOM2.

To bridge CMT with slab and hole parameters, we note that the basic waveguide dispersions are similar to our previous $\mathrm{PhC}$ designs on InP. We use 2-D simulations with 2-D effective indexes in SoI, which are very similar, typically $n_{\text {eff }} \approx 2.8$. Standard 2-D supercell calculations give the mode positions for a given width, and help precisely establish the full design, e.g., the sequence of five widths needed to have $10 \mathrm{~nm}$ channel spacing, taking into account material and modal ( $\mathrm{slab}+\mathrm{PhC}$ waveguide) dispersions, period $(a=540 \mathrm{~nm})$, hole diameter $(d=345 \mathrm{~nm})$, etc. This choice of five $10 \mathrm{~nm}$ channels is further justified by the $\sim 60 \mathrm{~nm}$ bandwidth that can best be hoped from optimized surface coupler. Here, 1-D, single-polarization grating couplers will be used, but polarization diversity is known to be a realistic option since our previous work in the FUNFOX project [16].

We solve (1) with boundary conditions $F(0)=1$ and $H_{1}\left(z_{\max }\right)=H_{2}\left(z_{\max }\right)=0$ by standard methods. Fig. 3(a) shows the eigenvalue spectrum of the associated $3 \times 3 \omega$-dependent matrix with typical parameters. For the W5.x waveguides, and standard $\sim 35 \%$ air-filling fraction, a length of $40 a$ per section is well adapted. The coupling coefficient $\kappa_{12}$ and the leakage excess contained in $\alpha_{2}$ were adjusted to improve spectral output by trial and error.

The parameters taken in the simulation are those that were fed into the design of a large set of samples, among which a particular device with good performances is chosen to illustrate the positive result of our approach. The waveguide widths, counted between center of edge rows, were decreasing by $27 \mathrm{~nm}$ steps from 2735 to $2627 \mathrm{~nm}$ (five steps). The period was $520 \mathrm{~nm}$ and the air-filling factor was $40 \%$. These values are not fed in CMT as such: they help in determining the band structure (by 2-D plane-wave expansion), which, in turns, is used to determine the CMT band parameters, notably the MSB anticrossings. The effective index of the host (the silicon slab on silica) fed into the plane-wave expansion is $n=2.8$ (for TE polarization).

In Fig. 3(a), the coupled modes are seen to consist mainly of the two even/odd modes HOM1 \pm HOM2 that have a common interaction with FM. The losses of both coupled modes are thus about identical, averaging $\alpha_{1}$ and the four times larger $\alpha_{2}$. Only when it comes to the slow down due to the FM coupling do, the losses deviate from higher values, as occurs for each of the even/odd mode identically. For the FM, losses are very low and only the hybridization with the coupled even/odd modes brings in comparatively large losses. At these points, the eigenmodes are full hybrids of the three modes.

Fig. 3(b) and (c) shows the color maps of HOM1 and HOM2 intensities $\left|H_{i}(z)\right|^{2}$ versus longitudinal position in waveguide (abscissa) and frequency (ordinate). Starting from the right, 

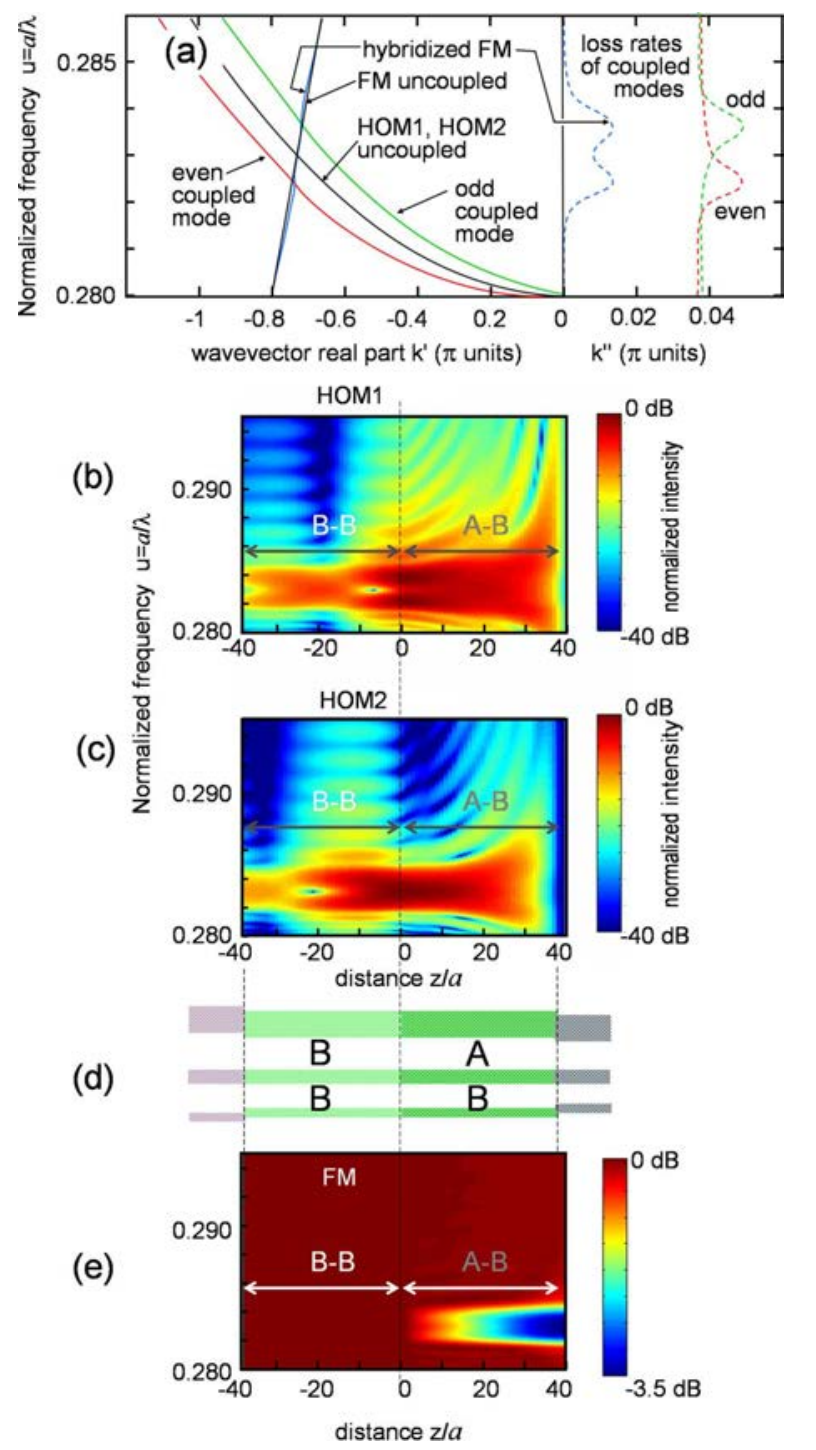

Fig. 3. (a) (Left) Dispersion of the three original modes (the steep FM and the two parabolic degenerate HOM1 and HOM2) superimposed on the dispersion relation of the coupled ones ("even" and "odd" are modes essentially consisting of the linear combinations HOM1 \pm HOM2, and the "hybridized FM" barely shifting away from the original FM). (Right) Losses of coupled modes (note the $\times 10$ abscissa factor). The even and odd modes always have high losses stemming from their slow nature. The FM mode becomes lossy only upon hybridization with HOM1 and HOM2. The slow down associated to the coupling also reinforces partly the two HOM losses; (b) color map of the HOM1 intensity as a function of position (abscissa) and normalized frequency (ordinate); (c) same for the HOM2 mode. Note the varying degree of observed splitting along the backward propagating HOM1 and HOM2 signals; (d) device reminder; (e) fundamental mode intensity, depleted only in the A-B MSB region.

backward propagation is quickly effective in restricting the useful frequencies of the HOM1 to the desired range. Then, the split behavior is progressively appearing for HOM1 in the region spanning from $15 a$ down to $-10 a$. The intensity of HOM2 grows later, as expected, and peaks at the $z=0$ transition at the central frequency, but slightly more backward if the integral over frequency is considered. The split behavior is then manifested mostly in the region from $z=-5 a$ down to $-25 a$.

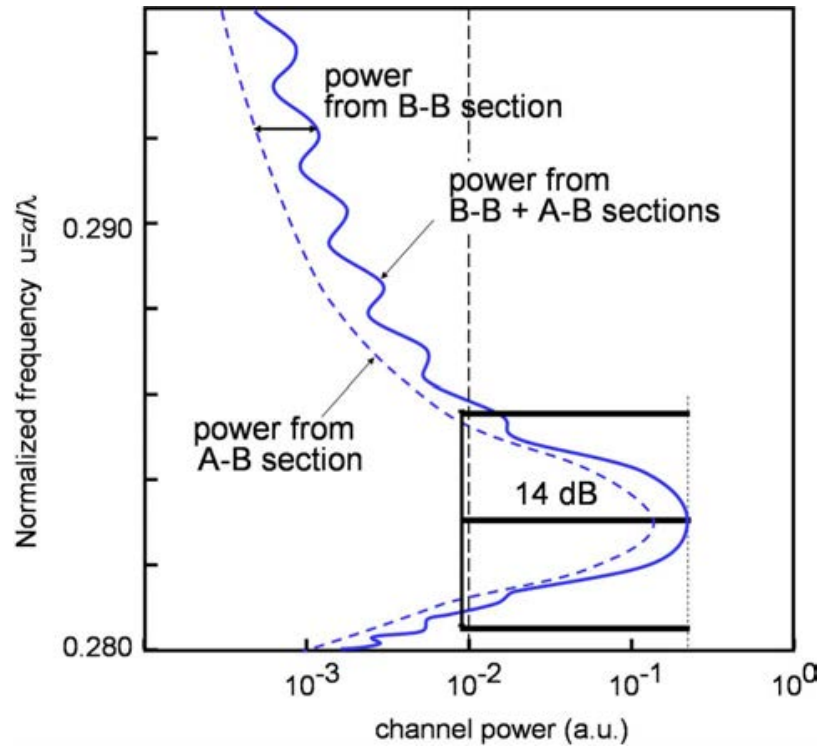

Fig. 4. Typical channel simulated power spectra with light either cumulated along the whole structure $(\mathrm{A}-\mathrm{B}+\mathrm{B}-\mathrm{B}$, solid line) or in only its $\mathrm{A}-\mathrm{B}$ section (dashed line); in this example, both sections are $40 a$ long.

The spectral response of the channel is primarily calculated as the power lost by HOM2 to the constant leakage, hence it follows the HOM2 trends. We plot in Fig. 4 the cumulated intensity from all $z$ values from $-40 a$ to $40 a$ (it is restricted to [ $-38 a$ to $38 a$ ] by trenches in the experiment, with weak consequences). The dashed line gives the cumulated power in the first spatial half, from $40 a$ down to 0 , while the solid line accounts for the whole power, until the boundary $-40 a$ whereby a more or less complete "cleanup" is expected. In total, a more flat-top filter shape is reached using the signal from the second half, with no strong effect on crosstalk. For the indicated bars, the crosstalk reaches $-15 \mathrm{~dB}$ on the good side and less $(\sim-11.5 \mathrm{~dB})$ on the bad side, allowing $-14 \mathrm{~dB}$ if the channel center is offset from its band center. The channel separation used here would correspond to $13 \mathrm{~nm}\left(\Delta u \approx 2.410^{-3}\right)$ without any group index correction. But in the SoI slab, the ratio of group effective index to phase effective index is $n_{g} / n_{\varphi}=1-\left(\lambda / n_{\varphi}\right)\left(\partial n_{\varphi} / \partial \lambda\right) \sim$ $1.2-1.3$. This dispersion is well known to result in shrinking spectral intervals, making this crosstalk prediction nearly valid for a $10 \mathrm{~nm}$ spacing.

Compared to the previous single-waveguide designs, the crosstalk is not doubled. However, some other benefit is there, such as the more top-hat line shape. More deeply, in this design, the fact that the light in the two HOMs does not sample both waveguides with the same longitudinal profile means that the dual cavity effect is partly ineffective. If the HOMs were closer to zero group velocity, the dual effect would remain: less backward spreading would cause the overall interaction to be more similar to that of a plane wave in a pair of coupled cavities. This would, however, complicate the design, which presently is based on simply width tuning, without a change in the period, hence an operation based on a stopband at a finite negative wavevector $\mathrm{k} / /$ and a slow, but nonzero group velocity. Operating at near-zero group velocity for the HOM 


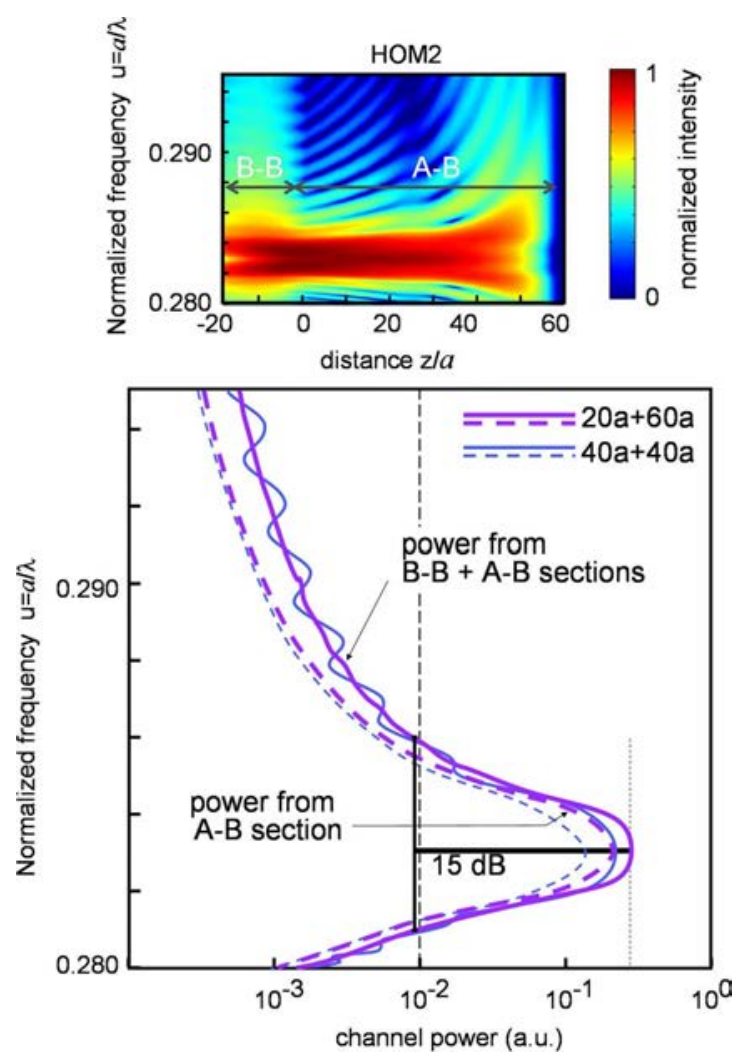

Fig. 5. (a) Color map of HOM2 intensity and (b) simulated spectra as in Figs. 3-4, but for a $20 a+60 a$ design, $20 a$ for the B-B section, and $60 a$ for the A-B section (thicker solid and dashed lines). The $40 a+40 a$ case is also reproduced (thinner solid and dashed lines) for comparison.

of all channels would imply, on the contrary, a tuning of the period, with guides of constant "Wx" type.

Causing more simultaneous interaction of HOM1 and HOM2 can be obtained in a $20 a+60 a$ configuration, because HOM1 is "refilled" by the FM in the added $20 a$ section. Correspondingly, running the same CMT simulation for this configuration, the results shown in Fig. 5 show a map with a more marked split behavior [see Fig. 5(a)]. The spectral channel output corresponds to the thicker lines of Fig. 5(b). Note the rather good "top-hat" shape of the resulting spectrum and the $\sim 1 \mathrm{~dB}$ larger output, a logical trend since the "harvesting" A-B section is a larger fraction of the device.

We wanted to confirm the operation of our design by more explicit but lengthy FDTD simulations. We kept the diminished length of the entrance B-B section to $20 a$ to assess the top-hat behavior, and we had enough computer power to model two consecutive channels. To get a better understanding of crosstalk and get more clear spectral signatures of leakage, we preferred a $18 \mathrm{~nm}$ spacing (without material or slab dispersion), namely 1569 and $1587 \mathrm{~nm}$. Our aim was therefore not to confirm CMT, but to give more physical clues.

Fig. 6 shows a typical screen capture illustrating the FDTD layout (We use OmniSim from PhotonDesign). Fig. 6(b) and (c) illustrates pictorially the spatial intensity distribution for nearly monochromatic excitation at the two-channel wavelengths. Here, five rows with identical hole diameters are used for separation, and three rows for final HOM2 leakage from the second (a)

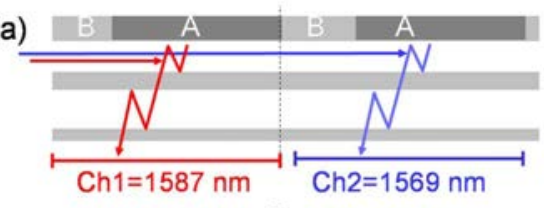

(b)

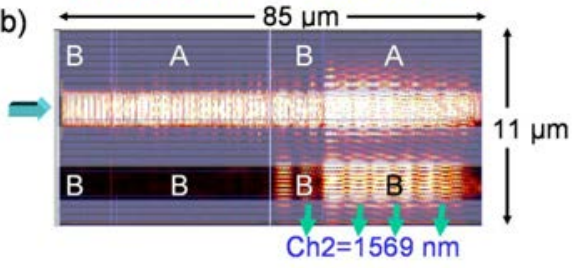

(c)

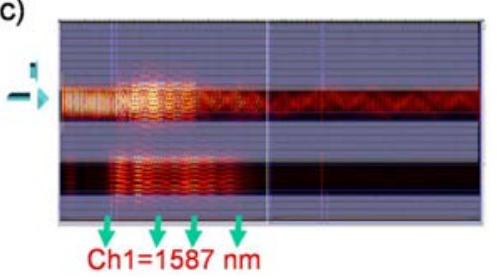

Fig. 6. Map of field intensities from FDTD calculations for the $40 a+40 a$ case; (a) scheme of the device operation; (b) pictorial field repartition for the $1569 \mathrm{~nm}$ channel; (c) pictorial field repartition for the $1587 \mathrm{~nm}$ channel. Stronger signals are whiter not calibrated.

guide to the collection channel. A guided wave is launched at the top left, with a mode profile calculated from a 1-D cut of the entrance waveguide. It corresponds well to the coupling from a ridge waveguide. The output signal for various levels of losses was recorded and integrated across the $80 a$ long channel output, for each of the two channels. Losses are here intended to account for out-of-plane losses. We therefore use the trick of the imaginary epsilon in air holes to mimic such out-of-plane losses, following the strategy we had applied with success in the "substrate" approach [24], [25]. It can be understood that because our waves do not imply ultimate confinement and lie above the light line of the photonic band diagram, the exact loss mechanism tends to be unimportant, only the fact that out-of-plane radiation occurs at air rows is important.

In Fig. 7(b), we present the various channel outputs $(\mathrm{Ch} 1$, $\mathrm{Ch} 2$, Bus2) for this FDTD calculation and, as can be located in Fig. 7(a), we also added monitoring of the internal power flux Bus1.

We perform these calculations based on losses that are variable from 0 to $1500 \mathrm{~cm}^{-1}$ in air (in air holes), by step of 500 $\mathrm{cm}^{-1}$. The two channels are centered at 1569 and $1587 \mathrm{~nm}$ in the constant index assumption of FDTD. This $18 \mathrm{~nm}$ difference was chosen here in the absence of "material" dispersion. At such a value, our current design could thus reach $20 \mathrm{~dB}$ crosstalk. Also note the signal asymmetry as predicted in CMT due to the parabolic shape of the HOM branch. We have lost, however, in FDTD, the modal decomposition of CMT. But the split behavior of each channel at low losses is clearly visible. It is seen that at losses of about $1000 \mathrm{~cm}^{-1}$, the split shape becomes a top-hat shape, with a $\sim 5 \mathrm{~dB}$ penalty however. This makes it clear that we have a good basic second-order design, but that refinements are needed to extract exactly a desired lineshape, e.g., flat-top, based on controlled coupling effects rather then based on losses. 


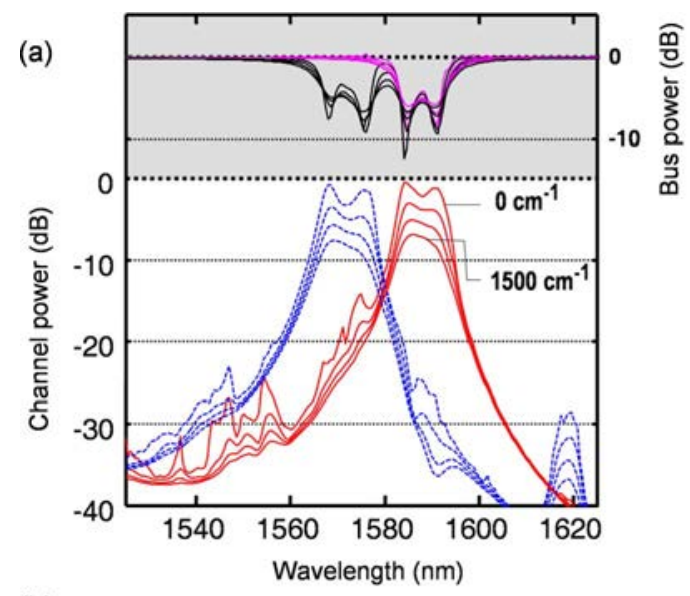

(b)

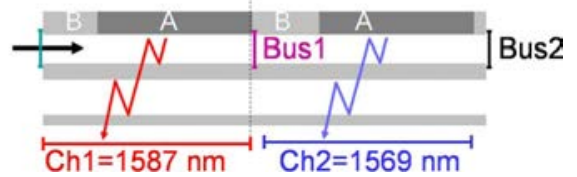

Fig. 7. FDTD calculations for the $20 a+60 a$ case; (a) power output of the two channels and fluxes intercepted across the top "bus" channel after the device for $\lambda=1569 \mathrm{~nm}$. The different curves correspond to different levels of imposed losses (usually in "air"), as shown; (b) scheme of device operation with FDTD "exciter" and detectors locations.

The good news that can be drawn from this set of simulations are twofold: 1) it confirms the obtainment of adjustable lineshape around the square-top profile; 2) it confirms that the $\mathrm{B}-\mathrm{B} / \mathrm{A}-\mathrm{B}$ structure is sound and effective in terms of cascading channels rather tightly. This was more cumbersome to check with CMT [10], on one hand, because of the task of matching modes, but also because of the neglect of scattering at the waveguide width change: although this latter effect is fortunately weak for the fundamental mode, it is in principle felt more strongly by the HOM. However, no specific penalty could be attributed to this mismatch in the present simulation.

\section{FABRICATION}

Due to the proven technology developed at IMEC [8], [18] and based on the previous InP-based experience [14], we fabricated on SoI a set of test structures. The silicon thickness was $220 \mathrm{~nm}$, giving vertical effective indexes in the range of 2.8 . They comprise a grating coupler for near-vertical fiber input, a long taper to reduce lateral mode size to our $\mathrm{PhC}$ waveguide width $\sim 2500 \mathrm{~nm}$, the demux device itself, with its five exit channels oriented at the expected output angle, as defined by few-micrometer-wide trenches, and finally, a $90^{\circ}$ turn on the main bus to observe the "through" signal as easily as possible. Blank devices with only a strip waveguide are also inserted to check the operation of the grating couplers alone. This is pictured in Fig. 8(c).

As previously established in other SoI devices made in ePIXfab, the grating involves a shallow etch, whereas the photonic crystal and trenches involved a deep etching.

\section{EXPERIMENTAL RESULTS}

We measured the spectra using a tunable laser source of a few milliwatts and a broadband InGaAs photodiode. Side col-

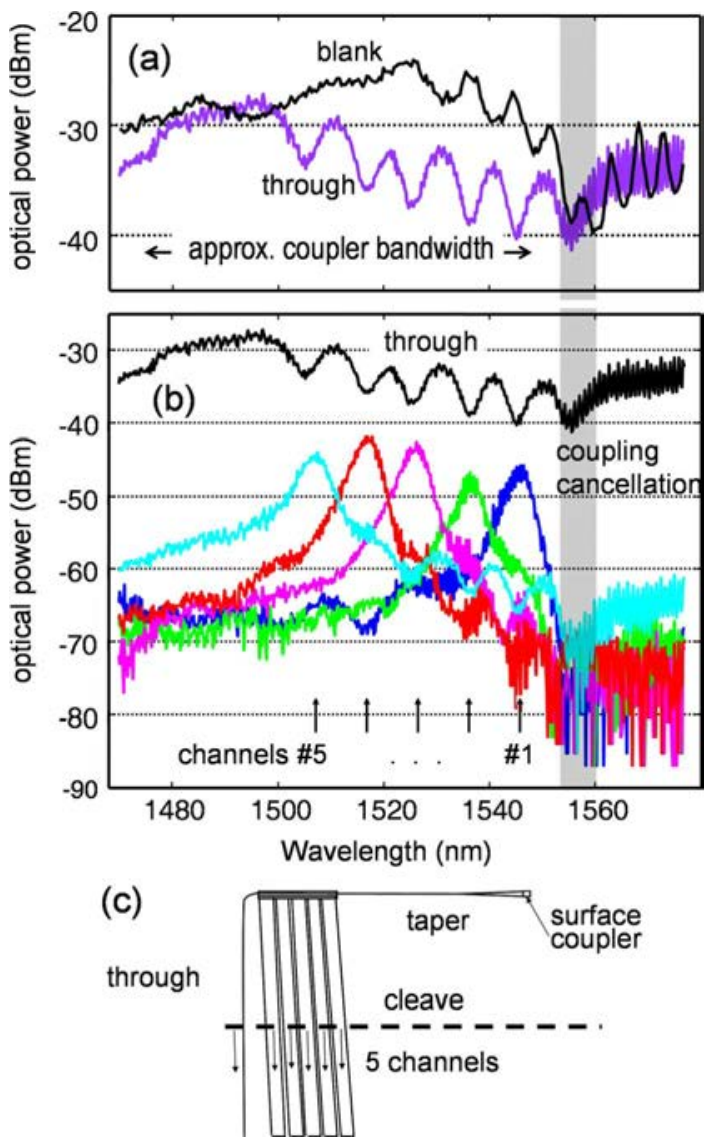

Fig. 8. Experimental raw results in one of the $40 a+40 a$ structures, as detailed in text: (a) transmission measurements of a blank system compared to a typical through channel. There are five output channels separated by $\Delta \lambda=10 \mathrm{~nm}$; note that the longest wavelength $1555 \mathrm{~nm}$ dip (gray area) probably arises from an incidental cancelation of the grating coupler's coupling efficiency; (b) spectral results of channel and "through" outputs; and (c) physical implementation mask.

lection at the cleaved edge was assessed by a camera before photocurrent measurements. A dark current of $1 \mathrm{nA}$ had to be subtracted with a $50 \mathrm{pA}$ fluctuation due to thermal drifts during the longer scans. This affects the accuracy of the measurements in their low-signal tails, but fortunately not in the top $20 \mathrm{~dB}$ range (photocurrents $>100 \mu \mathrm{A}$ ).

Devices with variable strength of intermediate spacer and final barrier were measured. We intend to give a more complete account elsewhere about the trends in terms of linewidth and rejection versus barrier parameters. We focus here on a structure with $5+3$ rows, with the two edge rows of the intermediate five rows barrier intentionally fabricated with smaller holes [see Fig. 2(b)], nearly $300 \mathrm{~nm}$ instead of $345 \mathrm{~nm}$ diameter, the basic $\mathrm{PhC}$ period being $520 \mathrm{~nm}$.

In Fig. 8(a), we show the blank waveguide data and the through channel port. The dips associated with each section demultiplexing action are clearly visible. The optimal coupling is situated around $1525 \mathrm{~nm}$. The dip at about $1555 \mathrm{~nm}$ seems to be a feature of the couplers themselves, possibly due to variable aspect ratio along the grating or a slightly over tilted fiber axis; it was seen systematically. It is thus likely to be responsible for the rightmost dip also in the through channel, whereas, as will be seen, the five other dips (the five left ones) being, as said, associated to the desired demux action. The input and output 
coupling losses are probably in the range of $10 \mathrm{~dB}$ each here, but they varied by a few decibels across samples.

As for the channel output spectra, see Fig. 8(b), we preferred not to normalize the data at this stage, so that the overall bumpy trend of the channel peak signals can be taken as a signature of the coupler efficiency, except for Channel \#2 at $1537 \mathrm{~nm}$. This choice of not normalizing conversely underlines the applicability of the device as built in a system. The peaks are $10 \mathrm{~nm}$ spaced, which shows the good quality of the technology as the underlying hole shifts are around $16 \mathrm{~nm}$ only.

The channel spectral shapes are not clearly showing the expected flat-top or splitting, but they have a substantially larger crosstalk than the previous first-order ones [14], [15]. For this $10 \mathrm{~nm}$ spacing, the crosstalks are essentially in the range -12 to $-15 \mathrm{~dB}$, with the exception of the weaker $1537 \mathrm{~nm}$ channel (\#2 from the right). It is difficult to check that the peaks are asymmetric. The channel \#1 peak at $1547 \mathrm{~nm}$ might be truncated on the long wavelength side by the coupler dip.

Overall, the results convincingly demonstrate that a $10 \mathrm{~nm}$ channel demux can be built along this technological option, exploiting the MSBs of broad photonic crystal waveguides. The footprint of the demux part is about $40 \mu \mathrm{m} \times 10 \mu \mathrm{m}$ per channel, hence $200 \mu \mathrm{m} \times 10 \mu \mathrm{m}$ in the present case. Comparison with AWG or flat spectrometer is quite favorable at first sight, these latter solutions demanding typically $100 \mu \mathrm{m} \times 100$ $\mu \mathrm{m}$. We do not detail this comparison anymore since an equivalent effort on spectral performance would also be needed to confirm this advantage up to the device stage. The insertion losses remains to be analyzed in more detail, but having a modest performance on this feature has been common when switching to radically new technology options.

\section{CONCLUSION}

We have attempted to improve on an SoI platform the demultiplexing performances of the photonic-crystal-waveguidebased demultiplexer that we started studying in 2005, and whose first "membrane" version was recently demonstrated on an InPbased system (see [15]). To this end, we have studied theoretically the role of a second guide adjacent to the first one, in which the higher order mode is experiencing second-order filtering. CMT and FDTD modeling have been used to check the demux mechanism and assess its performances.

The parameters considered comprise the thickness of the barrier between the two guides, the length of a "noncoupling" zone before the active one that provides room to clean up the higher-order-modes, and the spacing of the channel. The general target is the crosstalk between channels. The possibility to get a fairly flat-top-type spectral response was demonstrated in these models, and the impact of losses investigated.

Finally, the measurement of an SoI-based first device with five 10-nm-spaced channels showed a clearly improved crosstalk performance compared to the previous designs, reaching -12 to $-15 \mathrm{~dB}$. The insertion losses are still large, but not so much considering the fact that this is the first realization of this principle on SoI.

\section{ACKNOWLEDGMENT}

The authors would like to thank P. Dumon for his key role in the ePIXfab facility.

\section{REFERENCES}

[1] M. K. Smit and C. van Dam, "PHASAR-based WDM devices, principles, design, and applications," IEEE J. Sel. Topics Quantum Electron., vol. 2, no. 2, pp. 236-250, Jun. 1996.

[2] Y. Barbarin, X. J. M. Leijtens, E. A. J. M. Bente, C. M. Louzao, J. R. Kooiman, and M. K. Smit, "Extremely small AWG demultiplexer fabricated on InP by using a double-etch process," IEEE Photon. Technol. Lett., vol. 16, no. 11, pp. 2478-2480, Nov. 2004.

[3] B. Momeni, J. Huang, M. Soltani, M. Askari, S. Mohammadi, M Rakhshanderoo, and A. Adibi, "Compact wavelength demultiplexing using focusing negative index photonic crystal superprisms," Opt. Exp., vol. 14, pp. 2413-2422, 2006.

[4] D. Bernier, X. Le Roux, A. Lupu, D. Marris-Morini, L. Vivien, and E. Cassan, "Compact, low cross-talk CWDM demultiplexer using photonic crystal superprism," Opt. Exp., vol. 16, pp. 17209-17214, 2008.

[5] B. E. Little, S. T. Chu, P. P. Absil, J. V. Hryniewic, F. G. Johnson, F. Seiferth, D. Gill, V. Van, O. King, and M. Trakalo, "Very highorder microring resonator filters for WDM applications," IEEE Photon. Technol. Lett., vol. 16, no. 10, pp. 2263-2265, Oct. 2004.

[6] H. Takano, Y. Akahane, T. Asano, and S. Noda, "In-plane type channel-drop filter in a two-dimensional photonic crystal slab," Appl. Phys. Lett., vol. 84, pp. 2226-2228, 2004.

[7] F. Xia, L. Sekaric, and Y. A. Vlasov, "Ultracompact optical buffers on a silicon chip," Nature Photon., vol. 1, pp. 65-71, 2007.

[8] W. Bogaerts, P. Dumon, D. Van Thourhout, D. Taillaert, P. Jaenen, J. Wouters, S. Beckx, and R. Baets, "Compact wavelength-selective functions in silicon-on-insulator photonic wires," IEEE J. Sel. Topics Quantum Electron., vol. 12, no. 6, pp. 1394-1401, Nov./Dec. 2006.

[9] E. Viasnoff-Schwoob, C. Weisbuch, H. Benisty, C. Cuisin, E. Derouin, O. Drisse, G.-H. Duan, L. Legouézigou, O. Legouézigou, F. Pommereau, S. Golka, H. Heidrich, H. J. Hensel, and K. Janiak, "Compact wavelength monitoring by lateral outcoupling in wedged photonic crystal multimode waveguides," Appl. Phys. Lett., vol. 86, pp. 101107-1-101107-3, 2005

[10] L. Martinelli, H. Benisty, O. Khayam, G. H. Duan, H. Heidrich, and K. Janiak, "Analysis and optimization of compact demultiplexer monitor based on photonic crystal waveguide," J. Lightw. Technol., vol. 25, no. 9, pp. 2385-2394, Sep. 2007.

[11] L. Martinelli, H. Benisty, O. Drisse, E. Derouin, F. Pommereau, O Legouézigou, and G. H. Duan, "Impact of lithographic grid irregularity assessed on photonic crystal device selectivity," IEEE Photon. Technol. Lett., vol. 19, no. 5, pp. 282-284, Mar. 2007.

[12] M. Ayre, C. Cambournac, O. Khayam, H. Benisty, T. Stomeo, and T. F. Krauss, "Photonic crystal waveguides for coarse-selectivity devices," Photon. Nanostruct. Fundam. Appl., vol. 6, pp. 19-25, 2008.

[13] H. Hofmann, M. Kamp, A. Forchel, D. F. G. Gallagher, and H. Benisty, "Integrated wavelength monitoring in a photonic-crystal tunable laser diode," Photon. Nanostruct. Fundam. Appl., vol. 6, pp. 205-212, 2008.

[14] T. Stomeo, F. Van Laere, M. Ayre, C. Cambournac, H. Benisty, D. Van Thourhout, R. Baets, and T. F. Krauss, "Integration of grating couplers with a compact photonic crystal demultiplexer on an InP membrane,' Opt. Lett., vol. 33, pp. 884-886, 2008.

[15] F. Van Laere, T. Stomeo, C. Cambournac, M. Ayre, R. Brenot, H. Benisty, G. Roelkens, T. F. Krauss, D. Van Thourhout, and R. Baets, "Nanophotonic polarization diversity demultiplexer chip," J. Lightw. Technol., vol. 27, pp. 417-425, 2009.

[16] F. Van Laere, D. Van Thourhout, R. Baets, T. Stomeo, T. F. Krauss, M. Ayre, C. Cambournac, and H. Benisty, "Multifunctional photonic crystal compact demux-detector on InP," presented at the OFC/NFOEC 2008, San Diego, CA, Feb., Paper OThM5.

[17] H. Benisty, C. Cambournac, O. Khayam, M. Ayre, F. Van Laere, D. Van Thourhout, R. Baets, T. Stomeo, T. F. Krauss, M. Kamp, C. Hofmann, A. Forchel, and D. F. G. Gallagher, "Compact integrated photonic crystal demultiplexer for emitting and receiving InP photonic integrated circuits," presented at the ECOC 2008, Bruxelles, Belgium, Sep .

[18] P. Dumon, W. Bogaerts, R. Baets, J. M. Fedeli, and L. Fulbert, "Towards foundry approach for silicon photonics: Silicon photonics platform ePIXfab," Electron. Lett., vol. 45, pp. 581-582, 2009. 
[19] S. Olivier, M. Rattier, H. Benisty, C. J. M. Smith, R. M. De La Rue, T. F. Krauss, U. Oesterle, R. Houdré, and C. Weisbuch, "Mini stopbands of a one-dimensional system: The channel waveguide in a two-dimensional photonic crystal," Phys. Rev. B, Condens. Matter, vol. 63, no. 1-6, pp. 113311-, 2001.

[20] S. Olivier, H. Benisty, C. J. M. Smith, M. Rattier, C. Weisbuch, and T. F. Krauss, "Transmission properties of two-dimensional photonic crystal channel waveguides," Opt. Quantum Electron., vol. 34, pp. 171-181, 2002.

[21] S. Olivier, H. Benisty, C. Weisbuch, C. J. Smith, T. F. Krauss, and R. Houdré, "Coupled-mode theory and propagation losses in photonic crystal waveguides," Opt. Exp., vol. 11, pp. 1490-1496, 2003.

[22] S. Olivier, C. Weisbuch, and H. Benisty, "Compact and fault-tolerant photonic crystal add-drop filter," Opt. Lett., vol. 28, pp. 2246-2248, 2003.

[23] H. Benisty, "Modal analysis of optical guides with two-dimensional photonic bandgap boundaries," J. Appl. Phys., vol. 79, pp. 7483-7492, 1996.

[24] H. Benisty, D. Labilloy, C. Weisbuch, C. J. M. Smith, T. F. Krauss, A. Béraud, D. Cassagne, and C. Jouanin, "Radiation losses of waveguide-based two-dimensional photonic crystals: Positive role of the substrate," Appl. Phys. Lett., vol. 76, pp. 532-534, 2000.

[25] R. Ferrini, R. Houdré, H. Benisty, M. Qiu, and J. Moosburger, "Radiation losses in planar photonic crystals: Two-dimensional representation of hole depth and shape by an imaginary dielectric constant," J. Opt. Soc. Amer. B, Opt. Phys., vol. 20, pp. 469-478, 2003.

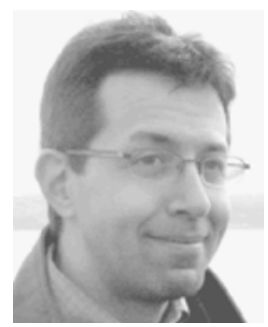

Henri Benisty (M'06) received the Ph.D. degree from the University Paris 6, Paris, France, in 1989.

$\mathrm{He}$ was involved in accumulation layers at $\mathrm{Si}$ interfaces. From 1982 to 1987, he was with the Ecole Normale Supérieure, France. His research topics have been first nanostructure growth and physics (Thomson, Orsay) and lamellar III-VI compounds (Paris 6 University, Paris, France). Since 1994, he has been involved in research on microcavities (mainly for LEDs) and in photonic crystals in two dimensions on III-V in Particulate Materials Center Laboratory, École Polytechnique, Palaiseau, France. He was with the University of Versailles till 2002. He is currently with the Laboratoire Charles Fabry de l'Institut d'Optique, Centre National de la Recherche Scientifique, Université Paris-Sud, Palaiseau, France. His research interests include applications of PhCs to LEDs, biophotonics, and miniature photonic ICs. He cofounded the biochip-related startup Genewave, Evry/Paris, France, in 2002.

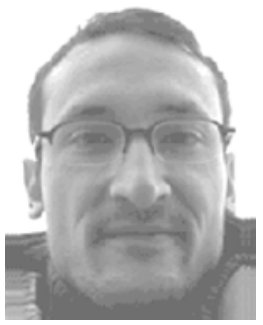

Cyril Cambournac was born in Saint-Mandé, France, in 1975. He received the B.S. degree in physics and the Ph.D. degree in engineering sciences from Franche-Comté University, Besançon, France, in 1998 and 2003, respectively.

From 2002 to 2005, he was with the Service d'Optique et d'Acoustique of the Applied Sciences Faculty, Free University of Brussels, Brussels, Belgium, as a Postdoctoral Fellowship, where he was engaged in studying nonlinear spatial optical phenomena, and particularly those related to soliton beams and instabilities in Kerr and non-Kerr media (photovoltaic crystals and liquid crystals). $\mathrm{He}$ is currently a Research Fellow at the Laboratoire Charles-Fabry de 1'Institut d'Optique, Centre National de la Recherche Scientifique, Université ParisSud, Palaiseau, France, where he is engaged in the experimental exploration of electromagnetic phenomena in linear and nonlinear photonic-crystal-type structures. His Ph.D. dissertation "Spatial Instabilities in Kerr Media: Arrays of Spatial Solitons and Symmetry Breaking of Multimode Solitons in a Planar Waveguide" was awarded the 2003 Saint-Gobain Young Researcher Prize from the French Physical Society.

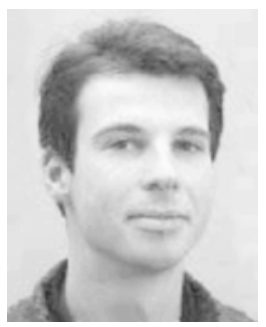

Frederik Van Laere (S'05) received the degree in electrical engineering and the Ph.D. degree in electrical engineering from Ghent University, Gent, Belgium, in 2004 and 2008, respectively.

$\mathrm{He}$ was engaged in design, fabrication and characterization of nanophotonic waveguide components and their integration with active functionality. Since 2008, he has been with Arcelor-Mittal, Gent.

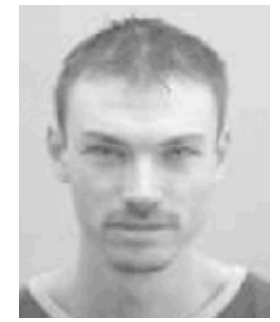

Dries Van Thourhout (M'98) received the physical engineering degree and the Ph.D. degree from Ghent University, Gent, Belgium, in 1995 and 2000, respectively.

From October 2000 to September 2002, he was with Lucent Technologies, Bell Laboratories, Murray Hill, NJ, where he was engaged in the design, processing, and characterization of InP/InGaAsP monolithically integrated devices. In October 2002, he joined the Department of Information Technology, Ghent University, where he is currently a member of the permanent staff of the Photonics Group. He is a Lecturer or Co-Lecturer for four courses within the Ghent University Master's in Photonics program. He is also coordinating the clean room activities of the research group. His research interests include the design, fabrication, and characterization of integrated photonic devices. Main topics involve silicon nanophotonic devices, heterogeneous integration of InP-on-Silicon, and integrated InP-based optical isolators. In addition, he is engaged on the development of new fabrication processes for photonic devices, e.g., based on focused ion beam etching and die-to-wafer bonding. He holds three patents, has authored and coauthored more than 60 journal papers, and has presented invited papers at several major conferences. 